\title{
Evaluation of compaction quality of earth-rock dam based on bacterial foraging-support vector regression algorithm
}

\author{
Jiajun Wang ${ }^{1, a}$, Denghua Zhong ${ }^{1}$ and Fei Wang ${ }^{2}$ \\ ${ }^{1}$ State Key Laboratory of Hydraulic Engineering Simulation and safety, Tianjin University, Tianjin 300072, China \\ ${ }^{2}$ State Key Laboratory of Hydraulic Engineering Simulation and safety, Tianjin University, Tianjin 300072, China
}

\begin{abstract}
Compaction quality is an important part of quality assessment of earth-rock dam. Current evaluation methods tend to use the graphical reports of compaction parameters based on real-time compaction monitoring system, or built compaction quality prediction model, such as the multiple linear regression model and the neural network model, to evaluate compaction quality of storehouse surface. However, the precision of these models is not enough. In this study the bacterial foraging -support vector regression algorithm is proposed and employed to establish a high prediction model between multiple attributes and compactness to evaluate compaction quality of storehouse surface. This compaction quality evaluation method gets rid of the one-sided shortcomings of the compaction graphical reports, and greatly improve the prediction accuracy compared with current models, which provides reasonable compaction quality evaluations for quality management works.
\end{abstract}

Keywords: evaluation of compaction quality, earth-rock dam, support vector regression, bacterial foraging algorithm.

\section{Introduction}

The compactness is an important indicator of compaction quality assessment of earth-rock dam. Currently, Researchers evaluate compaction quality mainly through two different ways, namely postevaluation such as sampling tests [1] and nucleon methods [2], and real-time evaluation which is based on the real-time monitoring system of earth-rock dam [3] [4]. However, given that postevaluation may incur compactness in dam filling process or affect the construction schedule. While the real-time monitoring system fails to evaluate compaction of storehouse surface directly. Therefore, some researchers have investigated the method of compaction quality assessment. Liu Donghai etc. establish the multiple linear regression model and neural network prediction model on the basis of the intrinsic relationship between rolling parameters and the compactness [5-7]. Furthermore, he adopted CVs to evaluate compaction quality, which is maturely used in the road construction [8]. However, during construction works, unsatisfactory grain composition of gravelly soil may produce strong noises to the vibration signal of rolling machine and therefore, there are inevitably deviations when the method is employed in the monitoring of the compaction. Liu Yvxi etc. [9], taking the climatic conditions of construction and integrating climatic data and compaction data, establish multiple linear regression model for compaction quality evaluation. The above researchers applied traditional method

${ }^{a}$ Corresponding author: jiajun_2014_bs@tju.edu.cn 
to establish the compaction quality prediction model, although they can guide the constructors to a certain extent, the reliability of their assessment outcomes is not enough due to the poor accuracy. In order to overcome the deficiencies of the above research, this study combining the bacteria foraging algorithm and the support vector regression algorithm, proposes bacterial foraging-support vector regression algorithm, and use this algorithm to reconstruct the compaction quality prediction model to evaluate the compaction quality of storehouse surface. The precision degree of this prediction model is improved by a large extend compared with multiple linear regression model and neutral network model.

\section{Bacterial foraging -support vector regression algorithm}

Support Vector Regression (SVR) is the employment of Support Vector Machine (SVM) in the domain of functions [10], which is mainly used in the approximation of functions. The concrete form of SVR can be determined only if proper penalty parameter $\mathrm{C}$, insensitive loss coefficient $\varepsilon$ and kernel function are chosen. It is unreasonable to carry out optimal choice for each parameter separately. Rather, the optimal parameter pairs $(\gamma, \varepsilon, \mathrm{C})$ shall be chosen simultaneously. Bacterial foraging algorithm[11], which simulate foraging behavior of Escherichia coli bacteria present in the human intestine, is adopt to optimize the hyper-parameters of the support vector regression algorithm in this work. The central idea of bacteria foraging -support vector regression algorithm runs as follows: take parameter pairs $(\gamma, \varepsilon, \mathrm{C})$ as the search space of bacteria and take the mean square error, which directly reflects the regression performance of SVR, as the fitness of bacteria. Then let bacteria move towards the optimal value by foraging behavior so as to find out the optimal positions of bacteria population and take them as the input parameter of SVR algorithm. Object-oriented thinking is adopted to write this algorithm and it is convenient to manage the dynamic changes of bacteria. The flowchart of the algorithm runs as Fig.1.

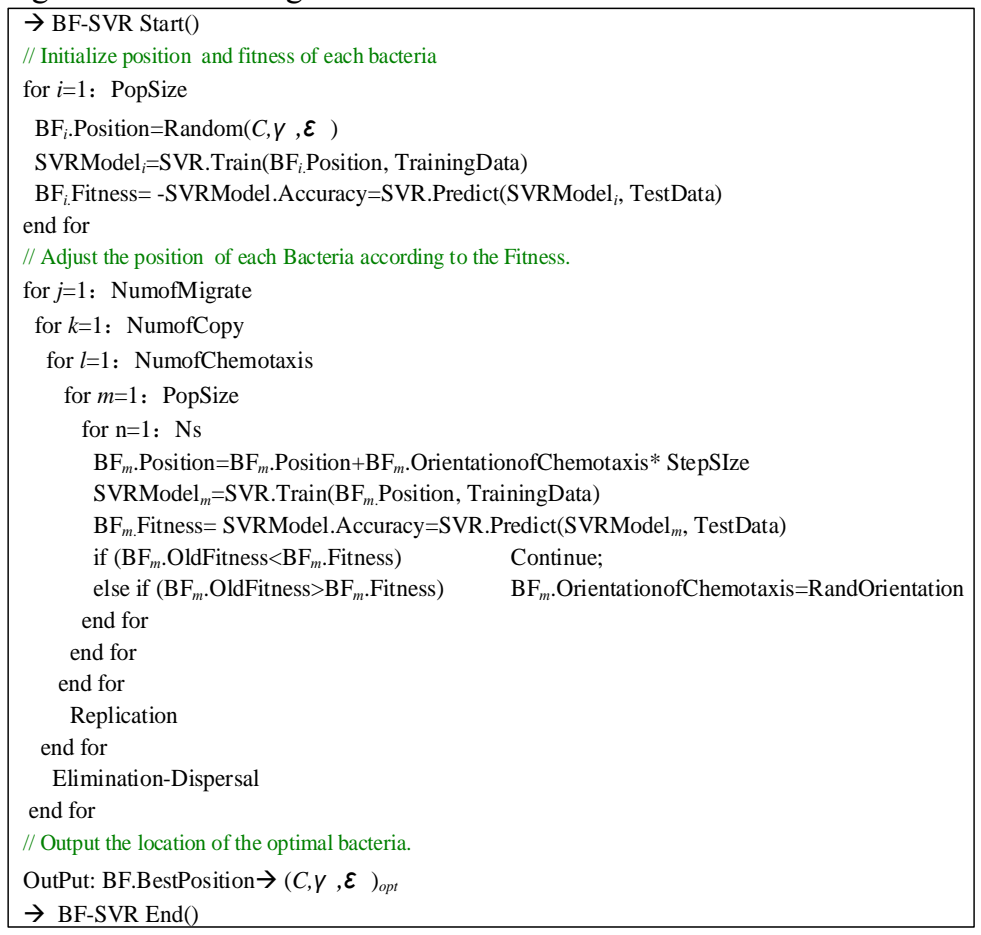

Figure 1. Pseudo code of BF-SVR algorithm process. 


\section{Evaluation of compaction quality of earth-rock dam based on bacterial foraging -support vector regression algorithm}

In order to establish high precision compaction quality prediction model, this study integrate the compaction data and material sources data as the basis of modeling data. This research takes the quality inspection data of gravel soil core wall from Aug. 2nd 2013 to Sept.10th 2015 as integration object, and 620 data are integrated in total. These data consist of so many attribute, in which some attributes are useful while the others may be redundant. Therefore, effective data reduction is essential. Finally, seven attributes of material source are chosen to evaluate the compactness of all materials, as is shown in the table 1 . Some of the data samples are presented in table 2.

Table 1. The Component of selected attributes.

\begin{tabular}{ccc}
\hline Category & Attribute & abbreviation \\
\hline \multirow{3}{*}{ Material sources parameters } & P5 content & P5C \\
& Maximum particle size & MPS \\
& All material moisture & AMMC \\
& content & HVP \\
& High vibration passes & SRP \\
Compaction parameters & Static rolling passes & RT \\
& Rolling thickness & RV \\
Quality test parameter & Rolling velocity & AMC \\
\hline
\end{tabular}

Table 2. A few samples of dataset used for constructing the model.

\begin{tabular}{ccccccccc}
\hline \multirow{2}{*}{ No. } & \multicolumn{7}{c}{ Input } & Output \\
\cline { 2 - 6 } \cline { 5 - 6 } & MPS[mm] & P5C[\%] & AMMC[\%] & RT[m] & HVP[1] & SRP[1] & RV[km/h] & AMC[\%] \\
\hline 1 & 115 & 37.5 & 10 & 0.29 & 13 & 5 & 2.36 & 99.2 \\
2 & 75 & 30.1 & 10.4 & 0.28 & 12 & 3 & 2.85 & 97.7 \\
3 & 126 & 43 & 10.5 & 0.35 & 10 & 3 & 2.23 & 100.2 \\
4 & 100 & 41.1 & 10.7 & 0.31 & 14 & 2 & 2.54 & 99.5 \\
5 & 170 & 47.6 & 8.8 & 0.25 & 13 & 3 & 2.63 & 98.2 \\
$\ldots$ & $\ldots$ & $\ldots$ & $\ldots$ & $\ldots$ & $\ldots$ & $\ldots$ & $\ldots$ & $\ldots$ \\
\hline
\end{tabular}

\subsection{Establishment of compaction quality prediction model based on bacterial foraging -support vector regression algorithm}

Based on the algorithm flowcharts in 1.3, we randomly allocate the 620 data we have integrated, partly used for training and partly for test in order to elevate the generalization of SVR algorithm and to eliminate over-fitting. Based on the research introduced by Üstün B etc. [12], parameters of SVR algorithm $\mathrm{C}=[1,108], \varepsilon=[0,0.2], \lambda=[0.01,2.0]$. And the size of the bacteria populations is set as 50 , while other parameters are set conventionally.

At the beginning of the algorithm, the bacteria are randomly distributed in the search space. Subsequently, with the iterations of the algorithm, bacteria move towards the optimal position. When the algorithm reaches its maximum number of iterations, the algorithm terminates and the position of optimal bacteria is the input parameter of the SVR algorithm. Fig.2 shows the initial positions of bacteria and Fig. 3 displays the movement of bacteria. 


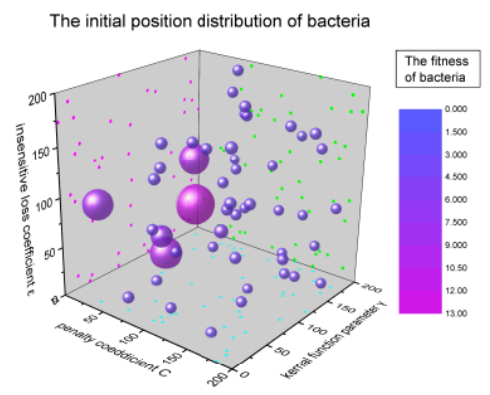

Figure 2. Initial position of bacteria.

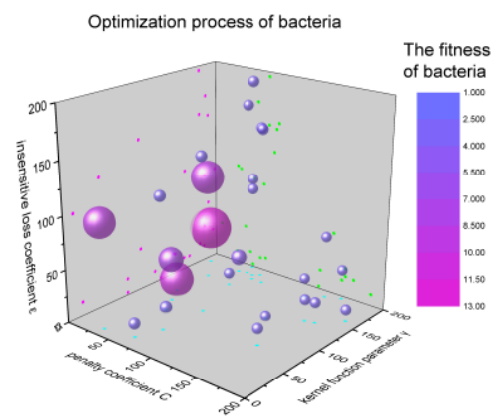

Figure 3. Position upgrading of bacteria.

Table 3. Error Statistic of Different Models.

\begin{tabular}{c|c|c|c}
\hline & linear regression model & Neural network model & BF-SVR model \\
\hline MSE & 2.855 & 2.122 & 0.685 \\
RAE & 2.083 & 1.9632 & 0.650 \\
MAE & 1.846 & 1.7452 & 0.576 \\
MA & 12.736 & 9.926 & 2.819 \\
X & 0.004 & & \\
MIN & & 0.015 & 0.004 \\
\hline
\end{tabular}

\subsection{Evaluation of storehouse surface compaction quality}

The monitoring data of ChangHe Dam, a large scale high core rock-fill dam in the southwest of China is taken for the evaluation of compaction quality. Firstly whole storehouse surface is partitioned with a $4 \mathrm{~m} \times 4 \mathrm{~m}$ lattice, and the average value of the data in each lattice is used to represent the input parameters, as is shown in Fig.4-5. Finally we taking the compaction quality prediction model constructed by the BF-SVR algorithm on the surface compaction quality prediction, the conclusions are shown in Fig.6. 


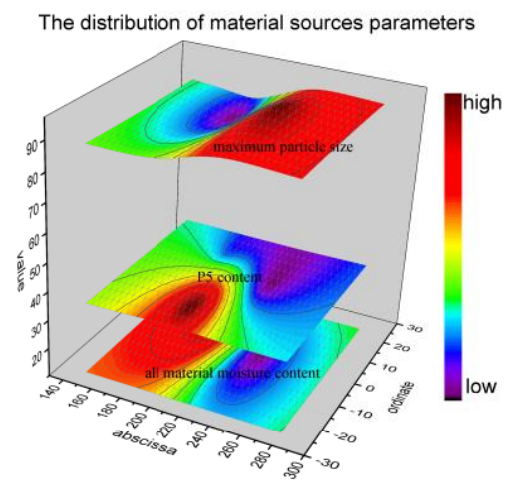

Figure 4. The distribution of material sources parameters.

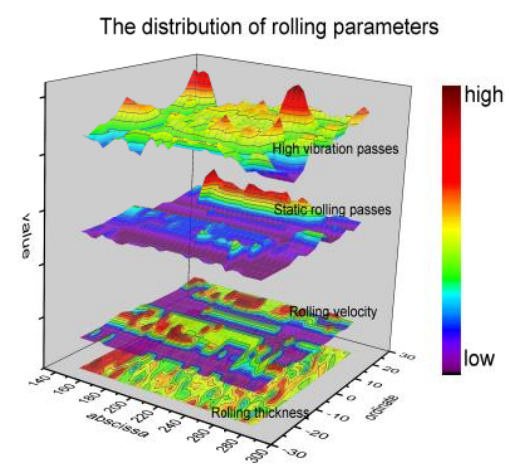

Figure 5. The distribution of rolling parameters.

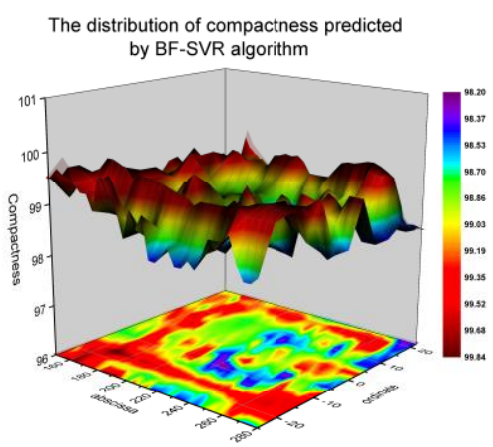

Figure 6. The distribution of compactness predicted by BF-SVR algorithm.

It can be seen from the Fig. 6 . that the percentage of the compaction quality of the surface above $98 \%$ is $100 \%$. That is to say, it conforms to construction design requirements and the surface quality reaches standards.

\section{Conclusion and prospect}

This research proposes bacteria foraging-support vector regression algorithm, and use it to build prediction model. The achievements of this research includes:

1. Bacteria foraging -support vector regression algorithm is proposed in this research, and compaction prediction model is reconstructed by this algorithm. Compared with multiple linear 
regression model and neutral network model, this new model has made much improvement in prediction precision.

2. When employed on the storehouse surface compaction quality prediction, this algorithm reveals the surface compaction quality more comprehensively, in comparison with traditional test of test pits and graphical reports of compaction parameters.

\section{Acknowledgments}

The authors wish to thank the Digital Dam project department of CHB hydroelectric station for providing the data necessary to complete this study. This research was funded by Innovative Research Groups of the National Natural Science Foundation of China (51321065), and the National Basic Research Program of China (973 Program) (2013CB035906)

\section{References}

1. CCS. DL / T 5129-2013 roller compacted embankment dam construction specifications [M]. China Electric Power Press, 2014. (In Chinese)

2. Zhao Xin. Nucleon density instrument application in earth dam compaction test [J]. Water Resources and Hydropower Construction, 2011 (6): 59-61. (In Chinese)

3. ZHONG, DengHua, LIU, et al. Real-time compaction quality monitoring of high core rockfill dam [J]. Science China Technological Sciences, 2011, 54(7):1906-1913.

4. Zhong D H, Cui B, Liu D H, et al. Theoretical research on construction quality real-time monitoring and system integration of core rockfill dam [J]. Science in China, 2009, 52(11):34063412.

5. Liu D, Sun J, Zhong D, et al. Compaction Quality Control of Earth-Rock Dam Construction Using Real-Time Field Operation Data[J]. Journal of Construction Engineering \& Management, 2012, 138(9):1085-1094.

6. Liu D, Li Z, Wang A. Correlation Analysis of Rolling Parameters and Real-Time Monitoring Index for Rockfill Dam Compaction Quality Evaluation [J]. Tianjin Daxue Xuebao, 2013, 46(4):361-366. (In Chinese)

7. Liu D H, Wang A G, Liu Y G, et al. Real-time monitoring and assessment of compaction quality for earth-rock dam basing on roller vibration behavior analysis[J]. Shuili Xuebao/journal of Hydraulic Engineering, 2014, 45(2):163-170.

8. Liu D, Li Z, Lian Z. Compaction quality assessment of earth-rock dam materials using rollerintegrated compaction monitoring technology. Automation in Construction, 2014, 44(8):234-246.

9. Liu Y, Zhong D, Cui B, et al. Study on real-time construction quality monitoring of storehouse surfaces for RCC dams [J]. Automation in Construction, 2014, 49:100-112.

10. Vapnik V N. An overview of statistical learning theory. [J]. IEEE Transactions on Neural Networks, 1999, 10(10):988-99.

11. Passino K M. Biomimicry of bacterial foraging for distributed optimization and control [J]. IEEE Control Systems, 2002, 22(3):52-67.

12. Üstün B, Melssen W J, Oudenhuijzen M, et al. Determination of optimal support vector regression parameters by genetic algorithms and simplex optimization. Anal Chim Acta [J]. Analytica Chimica Acta, 2005, 544(1-2):292-305. 